\title{
BOUNDS FOR NILPOTENT-BY-FINITE GROUPS IN CERTAIN VARIETIES
}

\author{
G. ENDIMIONI
}

(Received 18 May 1999; revised 11 October 2001)

\author{
Communicated by R. B. Howlett
}

\begin{abstract}
Let $\mathscr{B}_{e}, \mathscr{N}_{c}, \mathscr{N}$ and $\mathscr{F}$ denote respectively the variety of groups of exponent dividing $e$, the variety of nilpotent groups of class at most $c$, the class of nilpotent groups and the class of finite groups. It follows from a result due to Kargapolov and Čurkin and independently to Groves that in a variety not containing all metabelian groups, each polycyclic group $G$ belongs to $\mathscr{N} \mathscr{F}$. We show that $G$ is in fact in $\mathscr{N}_{c} \mathscr{F}$, where $c$ is an integer depending only on the variety. On the other hand, it is not always possible to find an integer $e$ (depending only on the variety) such that $G$ belongs to $\mathscr{N} \mathscr{B}_{e}$, but we characterize the varieties in which that is possible. In this case, there exists a function $f$ such that, if $G$ is $d$-generated, then $G \in \mathscr{N}_{f(d)} \mathscr{B}_{\text {e }}$. So, when $e=1$, we obtain an extension of Zel'manov's result about the restricted Burnside problem (as one might expect, this result is used in our proof). Finally, we show that the class of locally nilpotent groups of a variety $\mathscr{V}$ forms a variety if and only if $\mathscr{V} \cap \mathscr{N} \subseteq\left(\mathscr{N}_{\mathbb{C}^{\prime}} \mathscr{B}_{\ell^{\prime}}\right) \cap\left(\mathscr{B}_{\ell^{\prime}}, \mathscr{N}_{\mathrm{c}^{\prime}}\right)$ for some integers $c^{\prime}, e^{\prime}$.
\end{abstract}

2000 Mathematics subject classification: primary 20E10, $20 \mathrm{~F} 18$.

\section{Introduction}

If $c$ and $e$ are positive integers, we denote by $\mathscr{B}_{e}$ the variety of groups of exponent dividing $e$ and by $\mathscr{N}_{c}$ the variety of nilpotent groups of class at most $c$. Also, $\mathscr{N}=\bigcup_{c \geq 0} \mathscr{N}_{c}$ denotes the class of nilpotent groups and $\mathscr{F}$ denotes the class of finite groups. Recall that $\mathscr{F} \mathscr{N}$ is included in $\mathscr{N} \mathscr{F}$. More precisely, $\mathscr{F} \mathscr{N}_{c}$ is included in $\mathscr{N}_{c+1} \mathscr{F}$ for any $c \geq 0$. Indeed, if $G$ contains a finite normal subgroup $H$ such that $G / H \in \mathscr{N}_{c}$, it is easy to see that the centralizer of $H$ in $G$ has finite index and belongs to $\mathscr{N}_{c+1}$. Moreover, it is not difficult to show that any finitely generated group in $\mathscr{F} \mathscr{N}_{\text {c }}$ belongs to $\mathscr{N}_{c} \mathscr{F}[9$, Section 1.5].

(C) 2002 Australian Mathematical Society $1446 \cdot 7887 / 2000 \$ A 2.00+0.00$ 
Let $F$ be the free group of countably infinite rank. If $W$ is a subset of $F$, we denote by $\mathscr{V}(W)$ the variety of groups defined by the set of laws $w=1$, with $w \in W$.

First suppose that $W$ is not included in $F^{\prime \prime}$. By a result due to Kargapolov and Čurkin [10] and independently to Groves [8], for every integer $r \geq 0$, there exist integers $c, e$ depending on $\mathscr{V}(W)$ and $r$ such that each soluble group $G \in \mathscr{V}(W)$ with derived length $r$ belongs to $\mathscr{B}_{e} \mathscr{N}_{c} \mathscr{B}_{e}$. We do not know if $c$ and $e$ can be chosen depending on $\mathscr{V}(W)$ only (Proposition 2 and Theorem 2 below will give partial answers to this problem). Observe that each polycyclic group $G \in \mathscr{V}(W)$ is nilpotent-by-finite since $\mathscr{F} \mathscr{N} \subset \mathscr{N} \mathscr{F}$.

Conversely, consider a variety $\mathscr{V}(W)$ in which each polycyclic group is nilpotentby-finite. Since there exist metabelian polycyclic gloups which are not nilpotentby-finite, the variety of metabelian groups is not included in $\mathscr{V}(W)$; thus $W$ is not included in $F^{\prime \prime}$. So we have:

PROPOSITION 1. For a variety $\mathscr{V}(W)$ defined by a set of laws $w=1(w \in W)$, the following assertions are equivalent:

(i) each polycyclic group in $\mathscr{V}(W)$ belongs to $\mathscr{N} \mathscr{F}$;

(ii) $W \nsubseteq F^{\prime \prime}$.

This result leads to the following questions for a variety $\mathscr{V}(W)$ satisfying one of the previous assertions:

(1) Does there exist an integer $c$ such that each polycyclic group in $\mathscr{V}(W)$ belongs to $\mathscr{N}_{c} \mathscr{F}$ ?

(2) Does there exist an integer e such that each polycyclic group in $\mathscr{V}(W)$ belongs to $\mathscr{N} \mathscr{B}_{e}$ ?

In the next section we shall see that the first question has a positive answer (Theorem 1). It is not always the case for the second question but it is possible to characterize the varieties in which the answer is positive; furthermore, in this case, we shall show that each polycyclic group in $\mathscr{V}(W)$ belongs in fact to $\mathscr{N}_{c^{\prime}} \mathscr{B}_{e^{\prime}}$ for some integers $c^{\prime}, e^{\prime}$ (Theorem 2). Such bounds are global bounds, namely they are independant of the number of generators of considered groups. In Section 3 we shall see local bounds, that is, depending on the number of generators.

REMARK. Instead of question (2), one can put the following question (for a variety $\mathscr{V}(W)$ such that $\left.W \nsubseteq F^{\prime \prime}\right)$ :

$\left(2^{\prime}\right)$ Does there exist an integer $m$ such that each polycyclic group in $\mathscr{V}(W)$ belongs to $\mathscr{N}_{\mathcal{F}_{m}}$, where $\mathscr{F}_{m}$ is the class of finite groups of order at most $m$ ?

Suppose that such a $m$ exists and consider a polycyclic group $G \in \mathscr{V}(W)$. Denote by Fit $(G)$ the Fitting subgroup of $G$ and put $a=|G: \operatorname{Fit}(G)|$. For any integer $n>0$, 
the cartesian product $G^{n}=G \times \cdots \times G$ is polycyclic and belongs to $\mathscr{V}(W)$; thus $\left|G^{n}: \operatorname{Fit}\left(G^{n}\right)\right| \leq m$. Since Fit $\left(G^{n}\right)=\operatorname{Fit}(G)^{n}$, we obtain $a^{n} \leq m$ (for any integer $n>0$ ) and so $a=1$. Therefore, the previous question has a positive answer if and only if each polycyclic group in $\mathscr{V}(W)$ is nilpotent (and so we can take $m=1$ ).

\section{Global bounds}

Recall that an $n$-Engel group is a group satisfying the law $[x, n y]=1$, where $[x, n y]$ is defined by $[x, 0 y]=x$ and $[x, k y]=[[x, k-1 y], y]$ for all $k>0$, with $[x, y]=x^{-1} y^{-1} x y$. If $\Pi$ is a set of primes, a $\Pi$-free group is a group without elements of order $p \in \Pi$. We shall denote by $\gamma_{j}(G)$ the $j$ th term of the lower central series of a group $G$.

LEMMA 1. Let $\mathscr{V}(W)$ be a variety such that $W \nsubseteq F^{\prime \prime}$. Then there exist a finite set of prime numbers $\Pi$ and an integer $n$ (depending on the variety only) such that each $\Pi$-free nilpotent group in $\mathscr{V}(W)$ is $n$-Engel.

ProOF. By [6, Lemma 4], $\mathscr{V}(W)$ has a law of the form

$$
\left[x,_{m} y\right]^{e_{1}}\left[x,_{m+1} y\right]^{e_{2}} \cdots\left[x,_{m+k-1} y\right]^{e_{k}} v(x, y)=1 \quad\left(m, k \geq 1, v(x, y) \in F^{\prime \prime}\right),
$$

where the exponents $e_{1}, \ldots, e_{k}$ are not all zero (we may assume that $e_{1} \neq 0$ ). Let $\Pi$ be the set of prime numbers dividing $e_{1}$ and let $G$ be a 2 -generated $\Pi$-free nilpotent group in $\mathscr{V}(W)$. It suffices to prove that the nilpotency class of $G$ is bounded by an integer $n$ depending only on $\mathscr{V}(W)$. Let $\mu$ be the nilpotency class of $G$. Put $A=\gamma_{s}(G)$, where $s$ is the least integer $\geq(\mu+1) / 2$. Notice that $s<1+(\mu+1) / 2$ and that $A$ is abelian. For any $a \in A, y \in G$, we have

$$
\left[a,_{m} y\right]^{e_{1}}\left[a_{, m+1} y\right]^{e_{2}} \cdots[a, m+k-1, y]^{e_{k}} v(a, y)=1 .
$$

But $v(a, y)$ belongs to $\langle a, y\rangle^{\prime \prime}=\{1\}$, so

$$
\left[a,_{m} y\right]^{e_{1}}\left[a_{m+1} y\right]^{e_{2}} \cdots\left[a,_{m+k-1} y\right]^{e_{k}}=1 .
$$

Notice that $[a, m y]$ belongs to $\gamma_{m+s}(G)$. Now suppose that $\left[a,{ }_{m} y\right]^{r_{1}^{\prime}}$ belongs to $\gamma_{m+s+j}(G)$ for some integer $j \geq 0$. By raising to the $e_{1}^{j}$ th power, the previous relation gives

$$
\left[a,_{m} y\right]^{e_{1}^{j+1}}\left[a,_{m+1} y\right]^{e_{2} e_{1}^{j}} \ldots\left[a_{m+k-1} y\right]^{e_{1} e_{1}^{j}}=1 .
$$

Since $\left[a^{\prime e}, y\right]=\left[a^{\prime}, y\right]^{e}$ for any $a^{\prime} \in A$, we may write

$$
\left[a,,_{m} y\right]^{e_{1}^{+1}}\left[[a, m y]^{e_{1}^{\prime}}, y\right]^{e_{2}} \cdots\left[\left[a,,_{m} y\right]^{e_{1}{ }_{1}{ }_{k-1} y}\right]^{e_{k}}=1
$$


and so $[a, m y]^{e_{1}^{j+1}}$ belongs to $\gamma_{m+s+j+1}(G)$. Therefore, it follows by induction that $[a, m y]^{e_{1}}$ belongs to $\gamma_{m+s+j}(G)$ for any integer $j \geq 0$. By taking $j$ such that $m+$ $s+j>\mu$, we obtain $[a, m y]^{e_{1}^{j}}=1$ and so $[a, m y]=1$ since $G$ is $\Pi$-free. Hence, by [15, Proposition D], there exists an integer $t$ depending only on $m$ such that $\left[a, y_{1}, \ldots, y_{t}\right]=1$ for all $a \in A$ and all $y_{1}, \ldots, y_{t} \in G$. In particular, we have $\left[x_{1}, \ldots, x_{s}, y_{1}, \ldots, y_{t}\right]=1$ for all $x_{1}, \ldots, x_{s}, y_{1}, \ldots, y_{t} \in G$; thus $\mu+1 \leq s+t$. Since $s<1+(\mu+1) / 2$, we obtain $\mu<t+(\mu+1) / 2$ and so $\mu \leq 2 t$, as required.

Aplying Lemma 1 and $[3$, Corollary 1$]$ we may state

COROLlARY 1. Let $\mathscr{V}(W)$ be a variety such that $\longleftrightarrow \nsubseteq F^{\prime \prime}$. Then there exist a finite set of prime numbers $\Pi$ and an integer $c$ (depending on the variety only) such that each $\Pi$-free nilpotent group in $\mathscr{V}(W)$ belongs to $\mathscr{N}_{c}$.

As a consequence we obtain

PROPOSITION 2. Let $\mathscr{V}(W)$ be a variety such that $W \nsubseteq F^{\prime \prime}$. Then there exists an integer $c$ (depending on the variety only) such that each soluble group in $\mathscr{V}(W)$ belongs to $\mathscr{P} \mathscr{N}_{c} \mathscr{B}_{e}$, where $\mathscr{P}$ is the class of periodic groups and where $e$ is an integer depending on $\mathscr{V}(W)$ and on the derived length of $G$.

PROOF. Let $G$ be a soluble group in $\mathscr{V}(W)$. By the result of Kargapolov, Čurkin and Groves quoted above, there exist normal subgroups $K \unlhd H \unlhd G$ such that $K$ and $G / H$ belong to $\mathscr{B}_{e}$ (where $e$ depends on $\mathscr{V}(W)$ and on the derived length of $G$ ), $H / K$ being nilpotent. Denote by $L$ the normal subgroup of $H$ containing $K$ such that $L / K$ is the torsion subgroup of $H / K$; thus $H / L$ is a torsion-free nilpotent group. Hence, by Corollary $1, H / L$ belongs to $\mathscr{N}_{c}$, where $c$ is an integer depending only on $\mathscr{V}(W)$. Since $L$ is periodic, the proposition is established.

Now suppose that $G$ is polycyclic in the preceding proof. Then $L$ and $G / H$ are finite. Thus $H \in \mathscr{F} \mathscr{N}_{c}$ and so $H \in \mathscr{N}_{c} \mathscr{F}$ since any finitely generated group of $\mathscr{F} \mathscr{N}_{c}$ belongs to $\mathscr{N}_{c} \mathscr{F}\left[9\right.$, Section 1.5]. Therefore, $G$ is in $\mathscr{N}_{c} \mathscr{F}$ and we can state

THEOREM 1. Let $\mathscr{V}(W)$ be a variety such that $W \nsubseteq F^{\prime \prime}$. Then there exists an integer $c$ (depending on the variety only) such that each polycyclic group in $\mathscr{V}(W)$ belongs to $\mathscr{N}_{c} \mathscr{F}$.

Theorem 2 below shows that Theorem 1 fails if 'polycyclic' is replaced by 'finitely generated soluble'; in fact, Theorem 2 characterizes the varieties such that Theorem 1 remains true after this replacement. Before stating this theorem, we define the notion of efficient word, introduced by Black.

Let $F_{2}$ denote the free group of rank 2 generated by $x$ and $y$; this group will be considered as a subgroup of the free group $F$ of countably infinite rank. We shall write 
$\left\langle x^{F_{2}}\right\rangle$ (respectively $\left\langle y^{F_{2}}\right\rangle$ ) the normal closure of $\{x\}$ (respectively $\{y\}$ ) in $F_{2}$. Clearly, since $F_{2}^{\prime}=\left\langle x^{F_{2}}\right\rangle \cap\left\langle y^{F_{2}}\right\rangle$, each element $w \in F_{2}^{\prime}$ may be written in the forms

$$
w=w^{\prime} \prod_{i=r}^{i=s} y^{-i} x^{\alpha_{i}} y^{i}=w^{\prime \prime} \prod_{i=t}^{i=u} x^{-i} y^{\beta_{i}} x^{i} \quad\left(w^{\prime} \in\left\langle x^{F_{2}}\right\rangle^{\prime}, w^{\prime \prime} \in\left\langle y^{F_{2}}\right\rangle^{\prime}\right),
$$

where $r, s, t, u, \alpha_{i}, \beta_{j}$ are integers $(r \leq 0 \leq s, t \leq 0 \leq u)$. In [1], Black says that a word $w \in F_{2}^{\prime}$ is efficient if $w \notin\left\langle x^{F_{2}}\right\rangle^{\prime} \cap\left\langle y^{F_{2}}\right\rangle^{\prime}$ and if the set $\left\{\alpha_{r}, \ldots, \alpha_{s}, \beta_{t}, \ldots, \beta_{u}\right\}$ generates the ring of integers (that is, $\alpha_{r}, \ldots, \alpha_{s}, \beta_{t}, \ldots, \beta_{u}$ are coprime). Note that the first condition is redundant: if $w \in\left\langle x^{F_{2}}\right\rangle^{\prime} \cap\left\langle y^{F_{2}}\right\rangle^{\prime}$, it follows easily from the independence of left-normed basic commutators in $F_{2}^{\prime} / F_{2}^{\prime \prime}$ that $\alpha_{i}=\beta_{j}=0$ (for all $i, j$ ) and so $\alpha_{r}, \ldots, \alpha_{s}, \beta_{t}, \ldots, \beta_{u}$ cannot be coprime. The independence of left-normed basic commutators shows also the uniqueness of the expressions $w=$ $w^{\prime} \prod_{i=r}^{i=s} y^{-i} x^{\alpha_{i}} y^{i}\left(w^{\prime} \in\left\langle x^{F_{2}}\right\rangle^{\prime}\right)$ and $w=w^{\prime \prime} \prod_{i=t}^{i=u} x^{-i} y^{\beta_{i}} x^{i}\left(w^{\prime \prime} \in\left\langle y^{F_{2}}\right\rangle^{\prime}\right)$.

We shall say that $w=1$ is an efficient law if and only if $w$ is an efficient word. A characterization of varieties satisfying an efficient law is given by the following

PROPOSITION 3. For a variety $\mathscr{V}(W)$, the following assertions are equivalent:

(i) $W \nsubseteq F^{\prime p} F^{\prime \prime}$ for any prime $p$.

(ii) There exists in $\mathscr{V}(W)$ an efficient law.

PROOF. (i) implies (ii). By [6, Lemma 4], there exists in $\mathscr{V}(W)$ a law of the form

$$
w=[x, m y]^{e_{1}}\left[x,_{m+1} y\right]^{e_{2}} \cdots\left[x,_{m+k-1} y\right]^{e_{k}} v=1 \quad\left(m, k \geq 1, v \in F_{2}^{\prime \prime}\right),
$$

where the exponents $e_{1}, \ldots, e_{k}$ are coprime. In the usual way, consider $\left\langle x^{F_{2}}\right\rangle /\left\langle x^{F_{2}}\right\rangle^{\prime}$ as a module over the polynomial ring $\mathbb{Z}[T]$; namely, for $u \in\left\langle x^{F_{2}}\right\rangle$ and $P=\lambda_{n} T^{n}+$ $\cdots+\lambda_{1} T+\lambda_{0} \in \mathbb{Z}[T]$, we put

$$
P \bar{u}=\overline{y^{-n} u^{\lambda_{n}} y^{n} \cdots y^{-1} u^{\lambda_{1}} y u^{\lambda_{0}}}
$$

In particular, we have

$$
\bar{w}=\left(e_{1}(T-1)^{m}+e_{2}(T-1)^{m+1}+\cdots+e_{k}(T-1)^{m+k-1}\right) \bar{x} .
$$

The polynomial $Q=e_{1}(T-1)^{m}+e_{2}(T-1)^{m+1}+\cdots+e_{k}(T-1)^{m+k-1}$ may be written in the form

$$
Q=e_{m+k-1}^{\prime} T^{m+k-1}+\cdots+e_{1}^{\prime} T+e_{0}^{\prime},
$$

where $e_{m+k-1}^{\prime}, \ldots, e_{1}^{\prime}, e_{0}^{\prime}$ are coprime integers. In other words,

$$
w=y^{-(m+k-1)} x^{e_{m+k-1}^{\prime}} y^{m+k-1} \ldots y^{-1} x^{e_{1}^{\prime}} y x^{e_{0}^{\prime}} v^{\prime},
$$


with $v^{\prime} \in\left\langle x^{F_{2}}\right\rangle^{\prime}$. Clearly, it follows that $w$ is an efficient word.

(ii) implies (i). Suppose that $W \subseteq F^{\prime p} F^{\prime \prime}$ for some prime $p$. Since $F^{p} F^{\prime \prime}$ is a fully-invariant subgroup of $F$, we have $w \in F^{\prime p} F^{\prime \prime}$ for each law $w=1$ of $\mathscr{V}(W)$. Therefore, if $w \in F_{2}^{\prime}$, then we have necessarily $w=w^{\prime} \prod_{i=r}^{i=s} y^{-i} x^{\alpha_{i}} y^{i}$, with $w^{\prime} \in\left\langle x^{F_{2}}\right\rangle^{\prime}$ (respectively $w=w^{\prime \prime} \prod_{i=l}^{i=u} x^{-i} y^{\beta_{i}} x^{i}$, with $w^{\prime \prime} \in\left\langle y^{F_{2}}\right\rangle^{\prime}$ ), where $p$ divides each exponent $\alpha_{r}, \ldots, \alpha_{s}$ (respectively $\beta_{t}, \ldots, \beta_{u}$ ). Hence $w$ cannot be an efficient word.

REMARK. We have a similar result for a Milnor law, a concept introduced by Point [13]: $W \nsubseteq F^{\prime p} F^{\prime \prime}$ (for any prime $p$ ) if and only if there exists a Milnor law in $\mathscr{V}(W)$ [7, Proposition].

The next result gives various characterizations of varieties in which every polycyclic group is in $\mathscr{N} \mathscr{B}_{e}$, for some fixed integer $e$.

THEOREM 2. For a variety $\mathscr{V}(W)$, the following assertions are equivalent:

(i) There exists a positive integer e such that each polycyclic group in $\mathscr{V}(W)$ belongs to $\mathscr{N} \mathscr{B}_{e}$.

(ii) There exists a positive integer e such that each finitely generated soluble group in $\mathscr{V}(W)$ belongs to $\mathscr{N} \mathscr{B}_{e}$.

(iii) Each finitely generated soluble group in $\mathscr{V}(W)$ belongs to $\mathscr{N} \mathscr{F}$.

(iv) $W \nsubseteq F^{\prime p} F^{\prime \prime}$ for any prime $p$.

(v) There exist positive integers $c^{\prime}, e^{\prime}$ such that each soluble group in $\mathscr{V}(W)$ belongs to $\mathscr{N}_{c^{\prime}} \mathscr{B}_{e^{\prime}}$.

(vi) There exist positive integers $c^{\prime \prime}, e^{\prime \prime}$ such that each finite group in $\mathscr{V}(W)$ belongs to $\mathscr{N}_{c^{\prime}} \mathscr{B}_{e^{\prime \prime}}$.

PROOF. (i) implies (ii). This is a consequence of [12, Theorem 1].

(ii) implies (iii). This is obvious.

(iii) implies (iv). Suppose that $W$ is included in $F^{\prime p} F^{\prime \prime}$ for some prime $p$ and consider the restricted wreath product $G=(\mathbb{Z} / p \mathbb{Z}) ; \mathbb{Z}$. This group is metabelian and satisfies the law $[x, y]^{p}=1$; thus $G$ lies in $\mathscr{V}(W)$. Moreover, $G$ is finitely generated but $G$ is not nilpotent-by-finite (in fact, it is not polycyclic). Therefore $\mathscr{V}(W)$ does not satisfy (iii).

(iv) implies (v). [7, Proposition].

(v) implies (vi). In fact, we know that (iv) and (v) are equivalent [7, Proposition]. Hence we may assume that $W \nsubseteq F^{\prime p} F^{\prime \prime}$ for any prime $p$. By Proposition 3, there exists an efficient law in $\mathscr{V}(W)$ and so the result follows from a theorem of Black [1].

(vi) implies (i). Since a polycyclic group is residually finite, the implication is clear. 
Among the varieties satisfying some assertions of Theorem 2, one can quote the varieties defined by a non-trivial monoidal law, namely a law of the form

$$
u\left(x_{1}, \ldots, x_{n}\right)=v\left(x_{1}, \ldots, x_{n}\right),
$$

where $u\left(x_{1}, \ldots, x_{n}\right)$ and $v\left(x_{1}, \ldots, x_{n}\right)$ are distinct elements of the monoid freely generated by $x_{1}, \ldots, x_{n}$ (see [3] or [11]).

In general, the integers $e$ and $e^{\prime}$ occuring in Theorem 2 are distinct. In other words, if $G^{e}$ is nilpotent for each polycyclic group $G$ of a variety (for some fixed integer $e$ ), it is not always possible to bound the nilpotency class of $G^{e}$ by an integer $c$ depending on the variety only. For example, if $q$ is a prime-power, the variety $\mathscr{V}$ defined by the law $x^{q}=1$ satisfies the first assertion of Theorem 2 (with $e=1$ ); nevertheless, by Razmyslov's Theorem [16], there is no bound for the nilpotency class of finite groups of $\mathscr{V}$ if $q>3$.

However, as we shall see in the next section (Theorem 3), if $G$ is generated by $d$ elements, the nilpotency class of $G^{e}$ may be bounded by a function of $d$. When $e=1$, this question is clearly connected with the restricted Burnside problem.

\section{Local bounds and restricted Burnside problem}

In 1989, Zel'manov $[19,20]$ solved the restricted Burnside problem. More precisely, he proved that for any prime-power $q=p^{\alpha}$, there exists a function $f$ such that, for any positive integer $d$, the nilpotency class of every $d$-generated finite group satisfying the law $x^{q}=1$ is at most $f(d)$. By reduction theorems, the solution of the restricted Burnside problem follows, namely: up to isomorphism, for any fixed integers $d, e>0$, there are only a finite number of $d$-generated finite groups of exponent dividing $e$.

Zel'manov's proof also affords a solution of a problem posed in [18] (see [15, Proposition D]): for each positive integer $n$, there exists a function $f$ such that, for any positive integer $d$, the nilpotency class of every $d$-generated nilpotent $n$-Engel group is at most $f(d)$.

Notice that each polycyclic group satisfying the law $x^{q}=1$ (where $q$ is a primepower) or the law $[x, n y]=1$ is nilpotent (the second case is a consequence of a well-known result of Gruenberg [14, Result 12.3.3]).

More generally, consider a variety of groups $\mathscr{V}$ in which every polycyclic group is nilpotent. A finitely generated non-nilpotent soluble group has a finite non-nilpotent homomorphic image [14, Result 15.5.3]; hence each finitely generated soluble group of $\mathscr{V}$ is nilpotent (this also can be deduced from Theorem 2). So the previous statements lead to the following question: does there exist a function $f$ (depending on $\mathscr{V}$ ) such that, for any positive integer $d$, the nilpotency class of each $d$-generated soluble group of $\mathscr{V}$ is at most $f(d)$ ? 
As a consequence of Theorem 3 below, Corollary 2 will give an affirmative answer to this question.

THEOREM 3. Let e be a positive integer and let $\mathscr{V}$ be a variety of groups such that every polycyclic group of $\mathscr{V}$ is in $\mathscr{N} \mathscr{B}_{e}$. Then there exists a function $f$ such that, for any positive integer $d$, every $d$-generated soluble group of $\mathscr{V}$ belongs to $\mathscr{N}_{f(d)} \mathscr{B}_{e}$.

In order to prove this theorem, we need a preparatory lemma.

LEMMA 2. Let $e$ be a positive integer and let $\mathscr{V}$ be a variety of groups such that every polycyclic group of $\mathscr{V}$ is in $\mathscr{N} \mathscr{B}_{e}$. Then there exists a function $g$ such that, for any positive integer $d$, the derived length of every d-generated soluble group of $\mathscr{V}$ is at most $g(d)$.

ProOF. Consider a $d$-generated soluble group $G \in \mathscr{V}$. By Theorem 2, there exist positive integers $c^{\prime}, e^{\prime}$ depending on $\mathscr{V}$ only such that $G \in \mathscr{N}_{c^{\prime}} \mathscr{B}_{e^{\prime}}$. Hence $G$ contains a normal subgroup $H \in \mathscr{N}_{\mathfrak{c}^{\prime}}$ with $G / H \in \mathscr{B}_{e^{\prime}}$. By Zel'manov's solution of the restricted Burnside problem, the derived length of $G / H$ is bounded by an integer $g_{1}\left(d, e^{\prime}\right)$ (depending only on $d$ and $e^{\prime}$ ). Since the derived length of $H$ is bounded by $\left[\log _{2} c^{\prime}\right]+1$, the derived length of $G$ is at most $\left[\log _{2} c^{\prime}\right]+1+g_{1}\left(d, e^{\prime}\right)$.

PROOF OF THEOREM 3. Let $\mathscr{V}$ be a variety satisfying the hypothesis of the theorem and let $d$ be a positive integer. Let $\mathscr{R}_{g(d)}$ be the variety of all soluble groups of derived length at most $g(d)$, where $g$ is the function defined in Lemma 2. Consider the relatively free group $\Gamma_{d}$ of rank $d$ of the variety $\mathscr{V} \cap \mathscr{R}_{g(d)}$. It follows from Theorem 2 that $\Gamma_{d}$ belongs to $\mathscr{N} \mathscr{B}_{e}$; so there exists a positive integer $c$ such that $\Gamma_{d}$ belongs to the variety $\mathscr{N}_{c} \mathscr{B}_{e}$. By Lemma 2 , each $d$-generated soluble group $G \in \mathscr{V}$ lies in $\mathscr{V} \cap \mathscr{R}_{g(d)}$. Hence $G$ is a homomorphic image of $\Gamma_{d}$, and so $G \in \mathscr{N}_{c} \mathscr{B}_{e}$. Since $c$ depends only on $\mathscr{V}$ and $d$, the theorem is proved.

In the particular case where $e=1$, Theorem 3 yields

COROLlARY 2. Let $\mathscr{V}$ be a variety of groups in which every polycyclic group is nilpotent. Then there exists a function $f$ such that, for any positive integer $d$, every $d$-generated soluble group of $\mathscr{V}$ is nilpotent of class at most $f(d)$. In particular, there exists an integer $n$ depending only on $\mathscr{V}$ such that every soluble group of $\mathscr{V}$ is $n$-Engel (we can take $n=f(2)$ ).

In [4], Burns and Medvedev prove for each $n>0$ the existence of integers $c^{\prime}, e^{\prime}$ such that $\left(\mathscr{N}_{\ell^{\prime}} \mathscr{B}_{e^{\prime}}\right) \cap\left(\mathscr{B}_{e^{\prime}} \mathscr{N}_{\ell^{\prime}}\right)$ contains every $n$-Engel soluble group. Therefore, by using this result and the second part of Corollary 2 , we obtain 
COROLLARY 3. Let $\mathscr{V}$ be a variety of groups in which every polycyclic group is nilpotent. Then there exist positive integers $c^{\prime}, e^{\prime}$ such that every soluble group of $\mathscr{V}$ belongs to $\left(\mathscr{N}_{c^{\prime}} \mathscr{B}_{e^{\prime}}\right) \cap\left(\mathscr{B}_{e^{\prime}} \mathscr{N}_{\mathcal{C}^{\prime}}\right)$.

Notice that for a variety $\mathscr{V}$ and for an arbitrary positive integer $e$, the condition 'every finite soluble group of $\mathscr{V}$ is in $\mathscr{N} \mathscr{B}_{e}$ ' does not imply the condition 'every finite group of $\mathscr{V}$ is in $\mathscr{N} \mathscr{B}_{e}$ ' [5]. On the other hand, the conditions 'every finite soluble group of $\mathscr{V}$ is nilpotent' and 'every finite group of $\mathscr{V}$ is nilpotent' are equivalent: this follows from Schmidt's Theorem [14, Result 9.1.9]. Hence, as another consequence of Corollary 2, we have

COROLLARY 4. In a variety in which every polycyclic group is nilpotent, each finitely generated residually finite group is nilpotent.

This result may be considered as an extension of a theorem of Wilson [17], stating that each $n$-Engel finitely generated residually finite group is nilpotent.

\section{Locally nilpotent groups of a variety}

For convenience, denote by $\mathfrak{Y}_{e}$ the class of varieties in which every polycyclic group belongs to $\mathscr{N} \mathscr{B}_{e}$. Furthermore, let $\mathfrak{X}$ be the class of varieties $\mathscr{V}$ in which, for any positive integer $d$, the nilpotency class of $d$-generated nilpotent groups is bounded (the bound depending on $d$ and $\mathscr{V}$ only). It is easy to see that a variety $\mathscr{V}$ belongs to $\mathfrak{X}$ if and only if the class of locally nilpotent groups of $\mathscr{V}$ is a variety.

By Corollary $2, \mathfrak{X}$ contains $\mathfrak{Y}_{1}$. However, these classes are distinct: for example, if $m>1$ is a fixed odd integer, the dihedral group $D_{2 \times m}$ satisfies a law of the form $[x, 2 y]=[x, k y]$, with $k>2$ [2, Proposition 1]. Clearly, a nilpotent group satisfying this law is 2-Engel, and so of nilpotency class at most 3 [14, Result 12.3.6]. Thus the variety defined by the law $[x, 2 y]=[x, k y]$ belongs to $\mathfrak{X}$; but since the dihedral group $D_{2 \times m}$ is not nilpotent, this variety is not in $\mathfrak{Y}_{1}$.

Now consider a variety $\mathscr{V}$, defined by a set of laws $w=1(w \in W$, where $W$ is a subset of the free group $F$ ), and suppose that $W$ is included in $F^{\prime p} F^{\prime \prime}$ for some prime $p$. Since the wreath product $G=(\mathbb{Z} / p \mathbb{Z}) ?\left(\mathbb{Z} / p^{n} \mathbb{Z}\right)$ is a metabelian group satisfying the law $[x, y]^{p}=1$, this group is in $\mathscr{V}$ for all positive integers $n$. Moreover, $G$ is a 2 -generated nilpotent group of class $>p^{n}-1[17$, Result 2.2]. Hence $\mathscr{V}$ is not in $\mathfrak{X}$. In other words, we have proved that if $\mathscr{V}$ belongs to $\mathfrak{X}$, then $W$ is not included in $F^{\prime p} F^{\prime \prime}$ for any prime $p$; it follows from Theorem 2 that $\mathscr{V}$ belongs to $\mathfrak{Y}_{e}$ for some integer $e$. Thus $\mathfrak{X}$ is included in $\bigcup_{e>0} \mathfrak{Y}_{e}$. In fact, $\mathfrak{X}$ is a strictly smaller class than $\bigcup_{e>0} \mathfrak{Y}_{e}$. Indeed the variety $\mathscr{N}_{1} \mathscr{B}_{2}$ belongs to $\mathfrak{Y}_{2}$. This variety contains all dihedral groups, in particular $D_{2 \times 2^{n}}$. But since the nilpotency class of these groups is not bounded, the variety $\mathscr{N}_{1} \mathscr{B}_{2}$ is not in $\mathfrak{X}$. 
In short, we have $\mathfrak{Y}_{1} \subset \mathfrak{X} \subset \bigcup_{e>0} \mathfrak{Y}_{e}$, the inclusions being strict. The following result gives a characterization of varieties $\mathscr{V} \in \mathfrak{X}$

THEOREM 4. For a variety $\mathscr{V}$, the following assertions are equivalent:

(i) $\mathscr{V}$ belongs to $\mathfrak{X}$.

(ii) There exist positive integers $c^{\prime}, e^{\prime}$ (depending on $\mathscr{V}$ ) such that every nilpotent group of $\mathscr{V}$ belongs to $\left(\mathscr{N}_{\mathfrak{c}^{\prime}} \mathscr{B}_{e^{\prime}}\right) \cap\left(\mathscr{B}_{e^{\prime}} \mathscr{N}_{\mathcal{c}^{\prime}}\right)$.

Before proving this theorem, we establish two lemmas.

LEMMA 3. Let $x$ be a nilpotent element of a ring A. Suppose that for some integers $\alpha, \lambda, \beta_{0}, \ldots, \beta_{k}(\alpha, \lambda>0)$, we have the relations

$$
\alpha x^{\lambda}=\beta_{0} x^{\lambda}+\beta_{1} x^{\lambda+1}+\cdots+\beta_{k} x^{\lambda+k}=0 .
$$

Let $\alpha=p_{1}^{\epsilon_{1}} \ldots p_{t}^{\epsilon_{t}}$ be the factorization of $\alpha$ into a product of distinct prime powers and put $\epsilon=\epsilon_{1}+\cdots+\epsilon_{t}$. Then, if there exists an integer $j \in\{0, \ldots, k\}$ such that $\alpha$ and $\beta_{j}$ are coprime, we have $x^{\lambda+\epsilon k}=0$.

PROOF. The result is obvious if $\alpha=1$.

Suppose that $\alpha=p$ is prime (and so $\epsilon=1$ ). Let $j$ be the least integer of $\{0, \ldots, k\}$ such that $\alpha$ and $\beta_{j}$ are coprime. Thus we have the relation

$$
\beta_{j} x^{\lambda+j}+\beta_{j+1} x^{\lambda+j+1}+\cdots+\beta_{k} x^{\lambda+k}=0 .
$$

For any integer $\beta_{j}^{\prime}$ such that $\beta_{j} \beta_{j}^{\prime} \equiv 1(\bmod p)$, it follows that

$$
x^{\lambda+j}+\beta_{j+1} \beta_{j}^{\prime} x^{\lambda+j+1}+\cdots+\beta_{k} \beta_{j}^{\prime} x^{\lambda+k}=0 .
$$

By a standard argument, since $x$ is nilpotent, we obtain $x^{\lambda+j}=0$ and so $x^{\lambda+k}=0$.

Now suppose that $\epsilon>1$ and proceed by induction on $\epsilon$. Consider a prime $p$ dividing $\alpha$ and denote by $A_{p}$ the set of elements $a \in A$ such that $p a=0$. Clearly, $A_{p}$ is an ideal of $A$. Writing $\alpha^{\prime}=\alpha / p$, we have in the ring $A / A_{p}$ the relations:

$$
\alpha^{\prime} \bar{x}^{\lambda}=\beta_{0} \bar{x}^{\lambda}+\beta_{1} \bar{x}^{\lambda+1}+\cdots+\beta_{k} \bar{x}^{\lambda+k}=0 .
$$

It follows from the inductive hypothesis that the equality $\bar{x}^{\lambda+(\epsilon-1) k}=0$ holds in $A / A_{p}$. Therefore, we have in $A$ the relations

$$
p x^{\lambda+(\epsilon-1) k}=\beta_{0} x^{\lambda+(\epsilon-1) k}+\beta_{1} x^{\lambda+(\epsilon-1) k+1}+\cdots+\beta_{k} x^{\lambda+(\epsilon-1) k+k}=0 .
$$

Since in this case the result is established, we obtain $x^{\lambda+(\epsilon-1) k+k}=x^{\lambda+\epsilon k}=0$, as required. 
LEMMA 4. Let $c^{\prime}, e^{\prime}$ be positive integers. Then, there exists an integer $m=m\left(c^{\prime}, e^{\prime}\right)$ such that, for any nilpotent group $G \in\left(\mathscr{N}_{c^{\prime}} \mathscr{B}_{e^{\prime}}\right) \cap\left(\mathscr{B}_{e^{\prime}} \mathscr{N}_{c^{\prime}}\right)$ and for any $H \unlhd G$, we have $[h, m y] \in H^{\prime}$ for all $h \in H, y \in G$.

PROOF. Let $H$ be a normal subgroup of a nilpotent group $G \in\left(\mathscr{N}_{c^{\prime}} \mathscr{B}_{e^{\prime}}\right) \cap\left(\mathscr{B}_{e^{\prime}} \mathscr{N}_{c^{\prime}}\right)$. Denote by $A$ the ring of all endomorphisms of the abelian group $H / H^{\prime}$. For $y \in G$, the endomorphism $\hat{y} \in A$ is defined by $\hat{y}(\bar{h})=\overline{y^{-1} h y}(h \in H)$; thus $(\hat{y}-1)(\bar{h})=\overline{[h, y]}$. Notice that, $G$ being nilpotent, $\hat{y}-1$ is nilpotent. Moreover, since $G$ belongs to $\left(\mathscr{N}_{c^{\prime}} \mathscr{B}_{e^{\prime}}\right) \cap\left(\mathscr{B}_{e^{\prime}} \mathscr{N}_{c^{\prime}}\right)$, we have the relations $\left[h,{ }_{c^{\prime}+1} y^{e^{e^{\prime}}}\right]=\left[h,{ }_{c^{\prime}} y\right]^{e^{\prime}}=1$. Thus, we obtain in $A$ :

$$
\left(\hat{y}^{e^{\prime}}-1\right)^{c^{\prime}+1}=0=e^{\prime}(\hat{y}-1)^{c^{\prime}} .
$$

By using the relation $\left(\hat{y}^{e^{\prime}}-1\right)^{c^{\prime}+1}=\left(((\hat{y}-1)+1)^{e^{\prime}}-1\right)^{c^{\prime}+1}$, the first of these equations can be written in the form

$$
\beta_{0}(\hat{y}-1)^{c^{\prime}}+\beta_{1}(\hat{y}-1)^{c^{\prime}+1}+\cdots+\beta_{k}(\hat{y}-1)^{c^{\prime}+k}=0,
$$

where $k=e^{\prime}\left(c^{\prime}+1\right)-c^{\prime}$ and where $\beta_{0}, \ldots, \beta_{k}$ are integers, with $\beta_{k}=1$ (and $\left.\beta_{0}=0\right)$. Hence we may apply Lemma 3: there exists an integer $m=m\left(c^{\prime}, e^{\prime}\right)$ such that $(\hat{y}-1)^{m}=0$. In other words, $\left[h,_{m} y\right]$ belongs to $H^{\prime}$, as required.

ProOF OF THEOREM 4. (i) implies (ii). If $\mathscr{V}$ belongs to $\mathfrak{X}$, the nilpotency class of each 2-generated nilpotent group of $\mathscr{V}$ is bounded by an integer $n$. In particular, each nilpotent group of $\mathscr{V}$ is $n$-Engel. Hence the conclusion follows from the result of Burns and Medvedev [4] already used in the proof of Corollary 3.

(ii) implies (i). By [15, Proposition D], it is enough to prove that each nilpotent group of $\mathscr{V}$ is $n$-Engel for some integer $n$ depending only on $\mathscr{V}$. Consider a 2generated nilpotent group $G \in \mathscr{V}$. Since $G$ belongs to $\mathscr{N}_{\iota^{\prime}} \mathscr{B}_{e^{\prime}}$, the derived length of this group is bounded by an integer $r=r\left(c^{\prime}, e^{\prime}\right)$. Denote by $G^{(j)}$ the $j$ th term of the derived series of $G$ and apply Lemma 4; so there exists $m=m\left(c^{\prime}, e^{\prime}\right)$ such that $[h, m y] \in G^{(j+1)}$ for all $h \in G^{(j)}, y \in G$. From an immediate induction, it follows that $\left[x_{1+(r-1) m} y\right]$ belongs to $G^{(r)}$ for all $x, y \in G$, and so $\left[x_{1+(r-1) m} y\right]=1$. Therefore, each nilpotent group of $\mathscr{V}$ is $n$-Engel (for $n=1+(r-1) m$ ), and the theorem is proved.

\section{References}

[1] S. Black, 'Which words spell 'almost nilpotent'?', J. Algebra 221 (1999), 475-496.

[2] R. Brandl, 'On groups with small Engel depth', Bull. Austral. Math. Soc. 28 (1983), 101-110. 
[3] R. Burns, O. Macedońska and Y. Medvedev, 'Groups satisfying semigroup laws and nilpotent-byBurnside varieties', J. Algebra 195 (1997), 510-525.

[4] R. Burns and Y. Medvedev, 'A note on Engel groups and local nilpotence', J. Austral. Math. Soc. (Series A) 64 (1998), 92-100.

[5] O. Chapuis, 'Variétés de groupes et m-identités', C. R. Acad. Sci. Paris Sér. I Math. 316 (1993), 15-17.

[6] G. Endimioni, 'On the locally finite $p$-groups in certain varieties of groups', Quart. J. Math. Oxford 48 (1997), 169-178.

[7] - 'On conditions for a group to satisfy given laws', J. Group Theory 2 (1999), 191-198.

[8] J. R. J. Groves, 'On varieties of soluble groups. II', Bull. Austral. Math. Soc. 7 (1972), 437-441.

[9] P. Hall, 'Finite-by-nilpotent groups', Proc. Cambridge Philos. Soc. 52 (1956), 611-616.

[10] M. I. Kargapolov and V. A. Čurkin, 'Varieties of solvable groups', Algebra i Logika 10 (1971), 651-657.

[11] J. Lewin and T. Lewin, 'Semigroup laws in varieties of solvable groups', Proc. Cambridge Philos. Soc. 65 (1969), 1-9.

[12] F. Point, 'Conditions of quasi-nilpotency in certain varieties of groups', Comm. Algebra 22 (1994), 355-370.

[13] _ 'Milnor identities', Comm. Algebra 24 (1996), 3725-3744.

[14] D. J. S. Robinson, A course in the theory of groups (Springer, New York, 1982).

[15] A. Shalev, 'Combinatorial conditions in residually finite groups, II', J. Algebra 157 (1993), 51-62.

[16] M. Vaughan-Lee, The restricted Burnside problem (Clarendon Press, Oxford, 1990).

[17] J. S. Wilson, 'Two-generator conditions for residually finite groups', Bull. London Math. Soc. 23 (1991), 239-248.

[18] E. I. Zel'manov, 'On some problems of group theory and Lie algebras', Math. USSR Sbornik 66 (1990), 159-168.

[19] — 'Solution of the restricted Burnside problem for groups of odd exponent', Izv. Akad. Nauk. SSSR Ser. Mat. 54 (1990), 42-59.

[20] — 'Solution of the restricted Burnside problem for 2-groups', Mat. Sb. 182 (1991), 568-592.

\section{C.M.I.}

Université de Provence

UMR-CNRS 6632

39, rue F. Joliot-Curie

13453 Marseille Cedex 13

France

e-mail: endimion@gyptis.univ-mrs.fr 